\title{
BMJ Open Do women's empowerment and socioeconomic status predict the adequacy of antenatal care? A cross-sectional study in five South Asian countries
}

\author{
Asibul Islam Anik (10 , ${ }^{1}$ Md Rashedul Islam, ${ }^{2,3}$ Md Shafiur Rahman ${ }^{4,5}$
}

To cite: Anik Al, Islam MR, Rahman MS. Do women's empowerment and socioeconomic status predict the adequacy of antenatal care? A

cross-sectional study in five South Asian countries. BMJ Open 2021;11:e043940. doi:10.1136/ bmjopen-2020-043940

- Prepublication history and additional supplemental material for this paper are available online. To view these files, please visit the journal online (http://dx.doi.org/10.1136/ bmjopen-2020-043940).

Received 18 August 2020 Accepted 19 May 2021

Check for updates

(C) Author(s) (or their employer(s)) 2021. Re-use permitted under CC BY-NC. No commercial re-use. See rights and permissions. Published by BMJ.

For numbered affiliations see end of article.

Correspondence to

Asibul Islam Anik;

anikra93@gmail.com

\section{ABSTRACT}

Objectives Relative to the attention given to improving the measurement of adequacy of antenatal care (ANC) in South Asian (SA) region, the influence of women's empowerment and socioeconomic status (WESES) on adequate ANC services has hardly received any attention. This study aimed to investigate the present scenario of adequacy of ANC in SA and how its adequacy was associated with WESES.

Setting and participants Using the Demographic and Health Survey data set of five SA countries, that is, Afghanistan, Bangladesh, India, Nepal and Pakistan, 48 107 women were selected in this study who received at least one ANC component and had at least one live birth in the 3 or 5 years preceding the survey.

Analysis Multilevel logistic regression models were used to investigate the relationship between adequacy of ANC and WESES.

Results Only 30\% women received adequate ANC in SA, ranging from $8.4 \%(95 \% \mathrm{Cl} 7.1 \%$ to $9.9 \%)$ in Afghanistan to $39.8 \%$ (95\% Cl $37.4 \%$ to $42.2 \%$ ) in Nepal. The poor utilisation of adequate ANC services was most prevalent among the women residing in rural areas and that of poor families as well as low empowerment status in SA countries. Different levels of WESES, that is, highly empowered but poor (adjusted OR (AOR): $1.33 ; 95 \% \mathrm{Cl}$ 1.18 to 1.49 ), lowly empowered but rich (AOR: $2.07 ; 95 \%$ $\mathrm{Cl} 1.84$ to 2.32 ) and highly empowered and rich women (AOR: 3.07; 95\% Cl 2.75 to 3.43), showed significant positive association with adequate ANC services than the poor and low empowered women, after adjusting the potential covariates.

Conclusion As unsatisfactory level of adequate ANC services has been observed in SA region, this study suggests a nationwide comprehensive improvement of women's empowerment status as well as establishment of necessary healthcare centres in remote areas is essential to ensure long-term and sustainable adequacy of ANC services.

\section{INTRODUCTION}

Approximately 800 women die each day from complications in pregnancy and childbirth, and only South Asia (SA) accounts for about
Strengths and limitations of this study

- The approach of 'adequate ANC' estimation in this study allows the nationwide identification of women who received frequent, sufficient and appropriate care.

- This study can be used to show the existing gaps in coverage among different socioeconomic groups.

- This study considered the previous recommended antenatal care (ANC) visit (minimum four visits) rather than the WHO (2016) updated ANC guidance (minimum eight contacts), since Demographic and Health Survey data are only available for the previous recommendation.

$20 \%$ of the total maternal deaths worldwide. In $\mathrm{SA}$, while the maternal mortality ratio was 163 per 100000 live births in 2017, strategies like effective and adequate antenatal care (ANC) provision can accelerate the aim of achieving Sustainable Development Goal-3 in the area of maternal mortality (ie, reduction of global maternal death to less than 70 per 100000 live births) ${ }^{1-3}$ Most of the complications during pregnancy, like haemorrhage, eclampsia, sepsis, embolism, other pre-existing medical disorders, etc, which have been the leading causes of deaths and disabilities among women of childbearing age across the world, are preventable with adequate and cost-effective maternity care and counselling during and after pregnancy period. ${ }^{245}$ Evidence suggests that women's use of adequate ANC had a strong association with a lower occurrence of preterm births, and ANC alone can reduce maternal mortality by $20 \%$, given the good quality and regular attendance. ${ }^{67}$

Adequacy of ANC is a conditional process where women receive recommended ANC visits and components from skilled ANC 
providers. ${ }^{1}$ It could be measured according to four dimensions of the healthcare process: access to the care, that is, well timed (first visit during the first 3 months of pregnancy); sufficient (at least four antenatal visits); delivered by skilled health personnel; and received with recommended ANC packages or contents. ${ }^{178}$ But, in 2017, only half of the pregnant women in SA received the recommended minimum of four ANC visits, which is lower than in sub-Saharan Africa. ${ }^{9}$ On the other hand, the WHO recommended ANC packages include physical examinations (weight, height, blood pressure measurement, fetal heartbeat assessment), laboratory investigations (urine and blood samples), preventive procedures (tetanus injection and iron supplementation) and provision of information or counselling on signs of pregnancy complications. ${ }^{8}$ However, there are enormous disparities in receiving adequate $\mathrm{ANC}$ among women in different geographic areas and socioeconomic groups. ${ }^{10-12}$ Recommended ANC visits and receiving good quality ANC mostly depend on several community and individual-level factors such as geographic location, proximity to health centres, women's empowerment and household status. Previous studies reported that the likelihood of receiving good ANC was higher among women of rich household status in SA. ${ }^{10} 1113$ In particular, a previous study illustrated that highly empowered women from low and middle-income countries were positively associated with good quality maternal care during pregnancy. ${ }^{12}$ Nevertheless, women's educational level and earning status could not secure their healthcare decision-making autonomy because of the poor socioeconomic status (SES) of their households. ${ }^{14}$ So, there might be a hidden relationship of women's empowerment and socioeconomic status (WESES) (ie, lowly empowered but rich, highly empowered but poor, medium empowered but poor, etc) with the adequacy of ANC; however, such evidence in SA countries is lacking.

Most of the previous studies ${ }^{101113}$ were country specific and focused on either the contact coverage or on the content of ANC. However, none of those earlier studies comprehensively combined the conditional indices (ie, women who received recommended comprehensive care and coverage in all indicators) to measure and depict the whole picture of the adequacy of ANC services in SA. Thus, our study aimed to investigate the present scenario of the adequacy of ANC in SA and how adequacy is associated with WESES.

\section{METHODOLOGY}

\section{Data sources}

We used the nationally representative most recent Demographic and Health Survey (DHS) data from five SA countries-Afghanistan, Bangladesh, India, Nepal and Pakistan. The DHS surveys gather data on a range of population health indicators with a strong focus on maternal and child health in low and middle-income countries and comparable across countries. Women of reproductive age (15-49 years) without necessary ANC information and missing information on covariates were excluded from this study. Finally, 48107 women were used in this cross-sectional study who received at least one ANC component and had at least one live birth in the 3 or 5 years preceding the survey (online supplemental appendix table 1 ).

Regarding the ethical aspects of the DHS survey, details of administrative procedures, training, sampling strategies and methodology of DHS have been described elsewhere. ${ }^{1516}$ Because of the publicly available nature of DHS data with no identifying information, it was reviewed ${ }^{17}$ but exempted from ethics review approval by the Institutional Review Board at the first author's affiliation.

\section{Patient and public involvement}

Patients and the public were not involved in the development of research questions, design of the study, recruitment, and conduct of the study, or dissemination of the study results.

\section{Outcome variable}

The outcome variable of this study was the 'adequacy of antenatal care' which is the combination of (A) ANC coverage of at least one visit (ANC1), (B) intensity of ANC (ANC4), and (C) receipt of components of care. The ANC coverage (ANC1) was defined as the proportion of women who had at least one ANC visit with a skilled provider during their last pregnancy. ${ }^{78}$ Doctors, nurses, midwives and country-specific skilled health providers were considered as skilled ANC providers (online supplemental appendix table 2). The intensity of ANC (ANC4) was defined as four or more visits to ANC. Receipt of components of ANC was considered according to WHO recommendation ${ }^{19}$ and the availability of the item among all SA countries. Each respondent with at least one ANC visit was asked during the DHS survey whether she received specific care components during any of the consultations she attended during her pregnancy. Though the number of components collected varied across countries, we used four components (the measurement of blood pressure, urine test, blood test and information or counselling on pregnancy complications) which remained consistent across the countries included in this study. Since the components of ANC were assessed by looking at the proportion of women who received at least one ANC visit and at least one recommended component, we limited the analysis of components of ANC to women who received at least one ANC visit. Finally, a composite and dichotomised variable 'adequacy of ANC' was created by measuring the proportion of women who reported receiving all four components of ANC (checking blood pressure, giving a urine and blood sample and being counselled about pregnancy complications at any point during their pregnancy), received recommended intensity ANC of four or more visits and sought care from skilled ANC providers. The 'adequacy of ANC' was further coded as 1 (adequate ANC), and otherwise 0 . 


\section{Predictor variable}

The combinations of WESES have been considered as the predictor variable, where women's empowerment status (WES) was divided into three categories: low, medium and high; and their SES was divided into poor, middle and rich. Further, based on the intersectional axes of WES and SES categories, nine combinations of predictor variables were formed.

\section{Development of WESES}

The level of women's empowerment in the DHS is assessed using two indicators: (1) women's decision-making power in household-level decisions (access to healthcare, household purchasing and freedom to visit relatives), and (2) women's attitude towards violence. In addition to those, women's educational level, earning types and position in the household (household head or not) were included in this study to measure WES, as previous literature of the $\mathrm{SA}$ region had frequently studied these domains of measuring women's empowerment. ${ }^{20-22}$ This study considered the above-mentioned five indicators and the scoring process of these indicators was developed using evidence from previous literature to develop a composite index of $\mathrm{WES}^{21-24}$; where the higher values reflect the greater status of empowerment. The conceptualisation of women's empowerment (online supplemental appendix A) and the necessary coding for creating all five indicators have been narrated in the online supplemental appendix table 3. The indicator 'women's decision making power at household level' was constructed using three householdlevel decisions, that is, person who decides: respondent's healthcare, large household purchases and whether the respondent can visit her relatives. 'Women's attitude toward violence' was assessed using five variables describing whether beating was justified if the respondent: goes out without telling her husband, neglects children, argues with her husband, refuses sex with her husband and burns food. The indicator 'types of earning from employment' identifies whether respondents were employed or not, and if employed, this indicator includes the types of respondents' earning from their employment. The indicator 'educational level' includes the level of education of the respondents. And finally, 'headship of household' discloses whether the respondent herself was the household head or not.

Based on the scores of these five indicators, we performed principal component analysis (PCA) for each country to construct an overall empowerment index. During PCA, scree plots were analysed to define the number of components to be retained and orthogonal varimax rotation was applied to the retained components. We used the very first component only after loadings and getting scores of the components, and then the index scores were divided into quintiles (low, medium and high). Finally, the overall index of women's empowerment had been constructed for each selected country with three ordered categories: low, medium and high; where 'low' meant women have lower empowerment and 'high' meant women have higher empowerment. In the PCA analysis, the value of Kaiser-Meyer-Olkin measure of sampling adequacy ranged from 0.72 to 0.85 , indicated that the sample sizes of this study were adequate for PCA. The $\chi^{2}$ statistic in Bartlett's test of sphericity was significant (ie, $\mathrm{p}<0.001$ ) for all countries, which confirmed that the selected indicators of women's empowerment were intercorrelated. In addition, the reliability coefficient (Cronbach's alpha) score ranged from 0.60 to 0.79 , indicating an acceptable level of correlation among components. ${ }^{25}$

On the other hand, women's SES was measured based on the household wealth index, which was constructed based on housing characteristics and household assets. The detailed analytical procedures for measuring the wealth index in the DHS surveys are described elsewhere. $^{26}$ The SES for each country was categorised into five equal quintiles (poorest, poorer, middle, richer and richest), with the first representing the poorest $20 \%$, and the fifth representing the richest $20 \%$. In this study, the SES had further been divided into three classes, that is, poor (combining poorer and poorest), middle and rich (combining richer and richest).

\section{Controlling variables}

Following previous studies, ${ }^{1} 102728$ we included respondent's age (categorised as 15-24, 25-34 and 35-49 age groups), the number of living children (no child, 1-3 children, 4 and more children), media exposure (watching $\mathrm{TV}$, listening to radio and reading newspapers), modern contraceptive usage (yes, no), respondent's age at first birth (15-17, 18-46 years), wanted last pregnancy (yes, no), husband's educational level (no education, primary, and secondary and higher) and place of the region (urban, rural) as controlling variables in this study.

\section{Statistical analysis}

Descriptive statistics were used to estimate the proportion outcome with a $95 \%$ CI. In the pooled data set, individuals were nested within communities, and communities were further nested with countries. Thus, we used multilevel logistic regression models with a random intercept term at the community and country levels to investigate the relationship between adequacy of ANC and respondent's SES at the household level. A likelihood ratio test was performed to decide the choice of the multilevel model over the fixed-effect model. We presented both unadjusted OR and adjusted OR (AOR) along with 95\% CIs, as well as reported the significance level for the differences between the ORs of WESES. Furthermore, we performed sensitivity analysis to observe whether the incremental level of empowerment affects ANC coverage among the poor population. All the analyses considered sampling weight and the complex survey (DHS) design. For all analyses, $\mathrm{p}<0.05$ was set as the significant level. Multicollinearity was tested using variance inflation factor and observed no multicollinearity problem in the included models. Stata MP V.16.1 was used for all the analyses. 


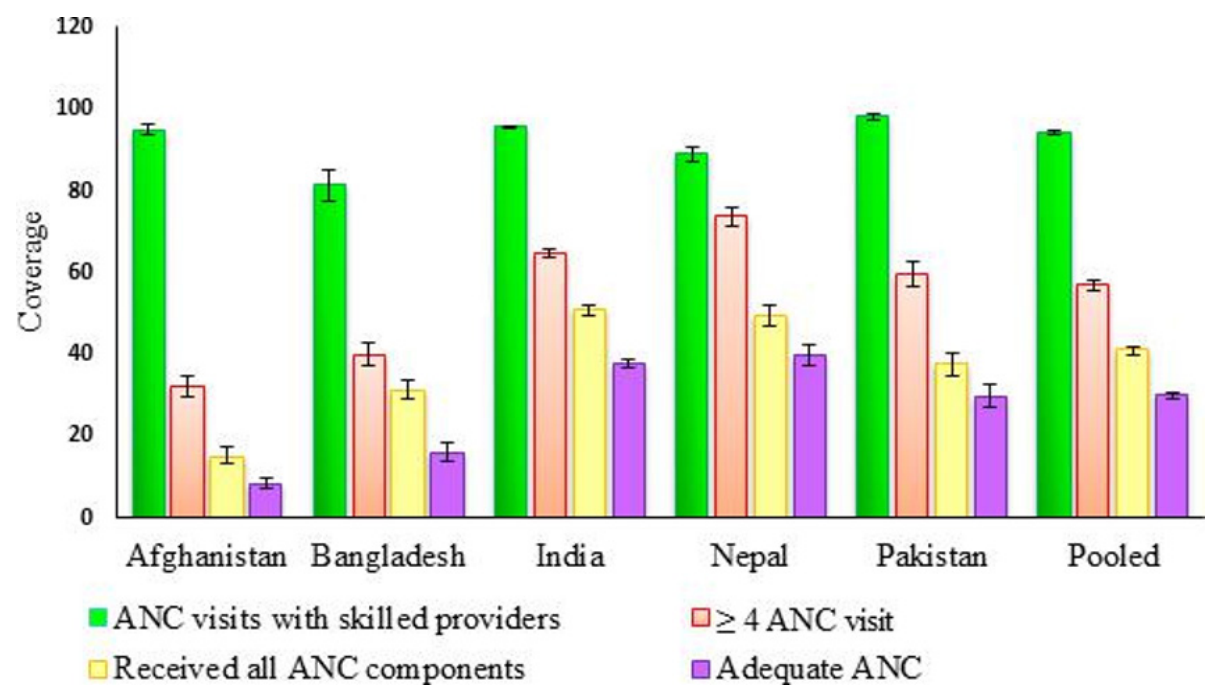

Figure 1 Overall coverage of antenatal care (ANC) in South Asia. Error bar presents 95\% Cls. Country-specific coverage has been tabulated in online supplemental appendix table 4.

\section{RESULT}

The overall coverage of ANC of SA countries has been shown in Figure 1. Three out of 10 women received adequate $\mathrm{ANC}$ in the $\mathrm{SA}$ region ranging from $8.4 \%$ in Afghanistan to $39.8 \%$ in Nepal. Though most of the SA women received at least one ANG visit from countryspecific skilled providers, their proportions decreased in terms of receiving four or more recommended ANC visits (about 57\%), ranging from $32.0 \%$ (in Afghanistan) to $73.7 \%$ (in Nepal), and components (about 41\%), ranging from $15.1 \%$ (in Afghanistan) to $50.8 \%$ (in India). Again, the practice of adequate ANC services was poor for the women residing in rural areas and that of poor families as well as low empowerment status in all SA countries (table 1).

The mean age $( \pm \mathrm{SD})$ of the respondents was 27.51 $( \pm 5.79)$ years. SA women who were both rich and highly empowered received about threefold more adequate ANC service $(44.24 \%$ vs $17.21 \%)$ than those of low empowered and poor (table 2). In addition, wives of higher educated husbands received comparatively more adequate ANC ( $37.37 \%$ vs $13.88 \%)$ than those women whose husbands were illiterate (online supplemental appendix table 5). On the other hand, the independent association of women's empowerment (online supplemental appendix table 6) and SES (online supplemental appendix table 7) with ANC showed that the coverage of ANC was significantly associated with the increment of empowerment and SES, individually.

The results of multilevel logistic regression models are presented in table 2 . The values of the likelihood ratio test were significant $(\mathrm{p}<0.000$ for model $\mathrm{I} ; \mathrm{p}<0.000$ for model II), meaning that random effects models are more appropriate for this study compared with the fixed-effect model. The unadjusted ORs from model I indicate that highly empowered but poor women had a $50 \%$ higher likelihood of receiving adequate ANC (OR: 1.50; 95\% CI 1.33 to 1.68 ) than the poor and lowly empowered women.
This likelihood for poor but highly empowered women decreased by $17 \%$ after adjusting all potential covariates, but still showed a significant and positive association with adequate ANC (AOR: 1.33; 95\% CI 1.18 to 1.49). Again, the unadjusted association of women's rich and high empowerment status (OR: 5.18; 95\% CI 4.68 to 5.72) showed more than fivefold increased odds than the poor and low empowered women while receiving adequate ANC in SA. However, the adjusted association shows that (model II) both highly empowered and rich and lowly empowered but rich women were 3.07 and 2.07 times more likely to receive adequate $\mathrm{ANC}$, respectively, than the lowly empowered and poor women.

Additionally, we investigated the significance level for the differences between the ORs of WESES from table 2. It was found that ANC coverage was less for the poor and low empowered women, compared with the higher empowerment and SES categories; and the difference between the ORs was statistically significant for almost all the WESES categories (online supplemental appendix table 8).

Since table 2 indicated that the probability of receiving adequate ANC was increasing proportionately with the increment of WESES, we further performed sensitivity analysis to investigate whether women's empowerment could accelerate the ANC coverage among low SES (online supplemental appendix table 9). The adjusted model (model II) in online supplemental appendix table 9 revealed that despite being low SES, the likelihood of receiving adequate $\mathrm{ANC}$ services was 1.36 times higher (AOR: $1.36 ; 95 \%$ CI 1.20 to 1.54 ) among the highly empowered women.

\section{DISCUSSION}

This study examined the adequacy of ANC in SA countries and how its adequacy was associated with WESES using nationally representatively recent household survey 
Table 1 Coverage of adequate antenatal care in South Asian countries according to the place of residence, socioeconomic status and level of empowerment $(n=48$ 107)

\begin{tabular}{|c|c|c|c|c|c|}
\hline & \multicolumn{5}{|c|}{ Coverage of adequate antenatal care $(95 \% \mathrm{CI})$} \\
\hline & $\begin{array}{l}\text { Afghanistan } \\
2015\end{array}$ & $\begin{array}{l}\text { Bangladesh } \\
2014\end{array}$ & $\begin{array}{l}\text { India } \\
\text { 2015-2016 }\end{array}$ & $\begin{array}{l}\text { Nepal } \\
2016\end{array}$ & $\begin{array}{l}\text { Pakistan } \\
2018\end{array}$ \\
\hline \multicolumn{6}{|l|}{ Place of residence } \\
\hline Rural & 5.9 (4.6 to 7.4$)$ & 11.3 (9.3 to 13.7$)$ & 33.7 (32.6 to 34.8$)$ & $30.6(27.3$ to 34.1$)$ & 21.1 (17.9 to 24.6$)$ \\
\hline Poor & 3.8 (2.8 to 5.2$)$ & 5.1 (3.7 to 7.0$)$ & 25.9 (24.4 to 27.4$)$ & 27.1 (24.3 to 30.0$)$ & 10.9 (8.9 to 13.3$)$ \\
\hline Middle & $7.4(5.5$ to 10.0$)$ & 9.7 (7.1 to 13.1$)$ & 39.5 (37.6 to 41.5$)$ & 35.3 (31.4 to 39.4$)$ & $23.8(20.0$ to 28.1$)$ \\
\hline Rich & $12.2(9.9$ to 14.9$)$ & 26.1 (23.1 to 29.4$)$ & 47.9 (46.2 to 49.5$)$ & 55.7 (52.3 to 59.1$)$ & 47.5 (44.2 to 50.8$)$ \\
\hline \multicolumn{6}{|l|}{ Empowerment level } \\
\hline \multicolumn{6}{|c|}{ Socioeconomic statusxempowerment level } \\
\hline \multicolumn{6}{|l|}{ Poor } \\
\hline Low empowered & 3.6 (2.5 to 5.3$)$ & 4.8 (3.11 to 7.4$)$ & 23.9 (21.7 to 26.2$)$ & 23.7 (19.8 to 28.0$)$ & 7.7 (5.6 to 10.4$)$ \\
\hline Medium empowered & 3.0 (1.6 to 5.6$)$ & 4.0 (2.3 to 6.8$)$ & 24.9 (22.8 to 27.1$)$ & 27.6 (23.3 to 32.2 ) & 11.3 (8.2 to 15.4$)$ \\
\hline Highly empowered & 5.4 (3.6 to 8.2 ) & 7.8 (4.6 to 13.0$)$ & 31.7 (28.9 to 34.6$)$ & 30.5 (26.2 to 35.1$)$ & 17.7 (12.6 to 24.4$)$ \\
\hline \multicolumn{6}{|l|}{ Middle class } \\
\hline Low empowered & 7.5 (5.1 to 10.8$)$ & 8.0 (4.3 to 14.5$)$ & 39.8 (36.9 to 42.9 ) & 28.8 (23.3 to 35.1$)$ & $16.0(11.1$ to 22.7$)$ \\
\hline Medium empowered & 5.4 (3.5 to 8.4 ) & 9.0 (5.6 to 14.3$)$ & 36.1 (33.1 to 39.3$)$ & 36.7 (29.9 to 44.0$)$ & 21.4 (16.8 to 26.9 ) \\
\hline Highly empowered & 9.5 (5.9 to 15.0$)$ & 11.9 (7.5 to 18.5$)$ & 42.7 (38.8 to 46.7$)$ & 44.4 (37.8 to 51.1$)$ & 34.7 (27.0 to 43.3$)$ \\
\hline
\end{tabular}

n, number of total respondents.

data from five SA countries. Our study has found that about $94 \%$ of women in $\mathrm{SA}$ received at least a single ANC visit and five out of 10 received at least four ANC visits. A recent UNICEF data (2019) on ANC showed that these statistics were far more downcast compared with the Middle East and North Africa (ANC4: 70\%), East Asia and the Pacific (ANC1: 96\%; ANC4: 75\%) and Latin America and the Caribbean (ANC1: 97\%; ANC4: 91\%). ${ }^{9}$ In $\mathrm{SA}$, the trend of seeking adequate ANC was not significant compared with other Asian regions. Within the last two decades, Indonesia, the Philippines, Vietnam and Cambodia showed more than a $10 \%$ increase in adequate ANC reception than Bangladesh, India and Nepal. ${ }^{29}$

In our study, inadequate ANC was mostly prevalent among poor and low empowered women who were as well residing in rural areas. Urban-rural differences were found to be greatest in other studies when overall use of ANC was low. ${ }^{29}{ }^{30}$ North Africa and West Africa, including Guinea, Mali, Niger and Senegal, had the most striking urban-rural gap (ie, three times as high) of receiving
ANC services, while countries of South/Southeast Asia possessed a considerable gap (about twice), especially in Bangladesh and Nepal. Physical remoteness from health facilities and inaccessibility of proper ANC services staying in rural areas were marked as the prime reasons for such disparities. ${ }^{30}$ Nevertheless, part of the explanation may lie in the impact of WESES on the use of ANC.

In low and middle-income countries, women's SES has been appeared to be a major determinant in terms of ensuring adequate ANC. And the poorest fifth of the population is less likely to have proper ANC than the richest fifth, mostly in Asia. ${ }^{10} 112730$ Whereas about half $(48 \%)$ of the SA population are multidimensionally poor, socioeconomic factors could impact the ANC of women in many ways. ${ }^{10} 31$ But, without empowering women multidimensionally along with the improvement of their SES, any further improvement of effective utilisation of maternal healthcare would not contribute to adequate ANC services for marginal groups. ${ }^{1027}$ Because household wealth status was found to be weakly associated with the 
Table 2 Association of women's empowerment and socioeconomic status with coverage of antenatal care in the pooled data $(n=48$ 107)

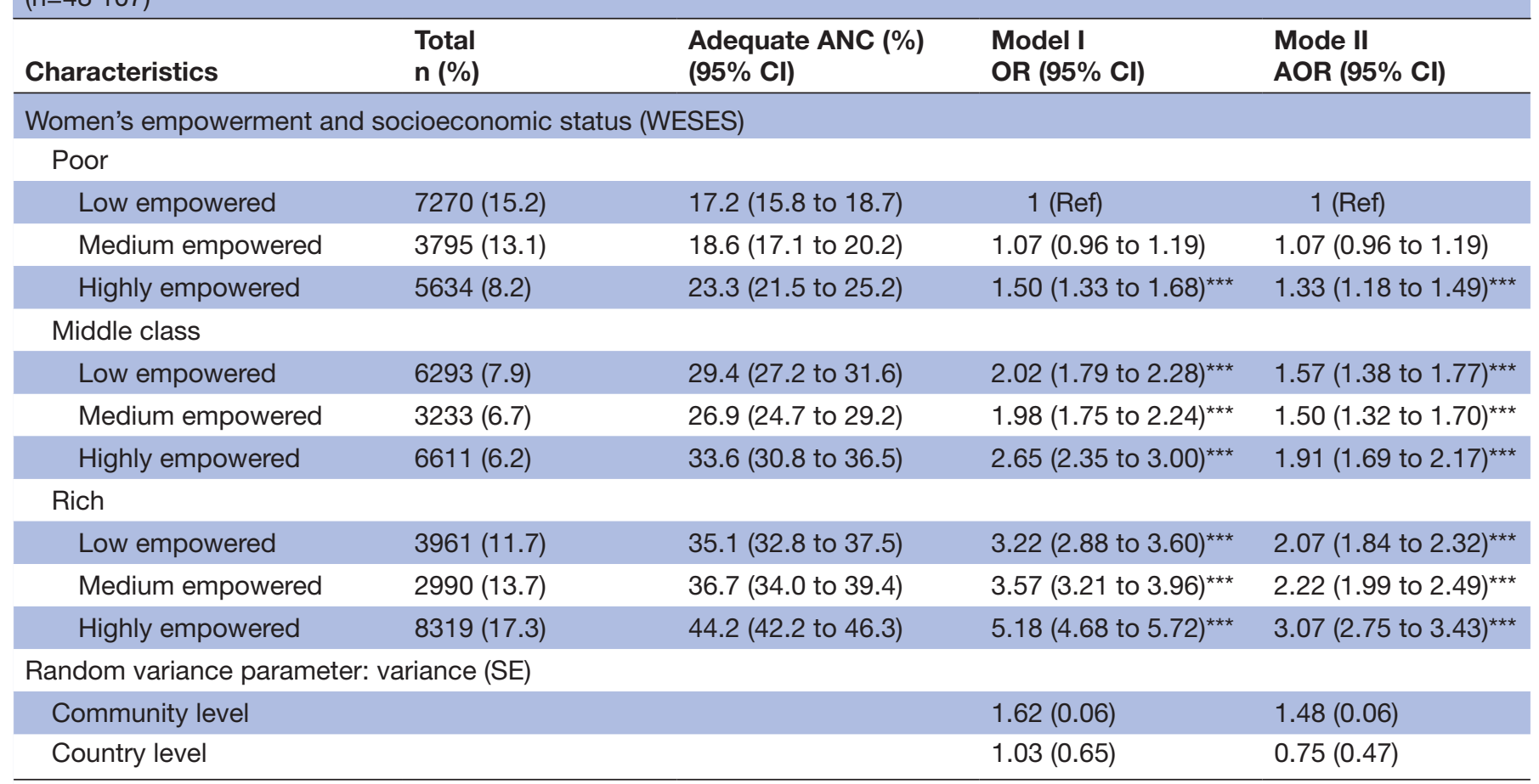

Model II was additionally adjusted by respondent's age, number of children, media exposure, modern contraceptive usage, age at birth, wanted last pregnancy, husband's educational level and place of region.

Significance level: ${ }^{\star} p<0.05 ;{ }^{* *} p<0.01 ;{ }^{* \star} p<0.001$.

Results of full models are presented in online supplemental appendix table 5.

ANC, antenatal care; AOR, adjusted OR; n, number of total respondents; Ref, reference category.

involvement in decision-making ${ }^{14}$; and lower decisionmaking power resulting from lower empowerment status consequently jeopardises proper maternal care during pregnancy. ${ }^{27}$ Therefore, lowly empowered women, even though residing in a wealthy and urban environment, might get inadequate ANC services due to active discrimination by the providers or due to women's low empowerment to demand higher quality services from the providers. ${ }^{32}$ In contrast, along with the outcome of the study from Guinea, ${ }^{33}$ this study revealed a positive and significant effect of women's empowerment on the utilisation of adequate ANC services, even among the poor people. In a nutshell, this study indicated women's higher empowerment status as a mitigating factor to the known risk of low SES on ANC coverage.

More specifically, our study revealed that women with higher education and empowerment status were more likely to use ANC services in any regional setting, which is matched with the outcomes of several studies. ${ }^{11} 272932$ Perhaps because educated women are more likely to realise the benefits of using maternal healthcare services. ${ }^{34}$ Again, education usually improves the empowerment level of women by increasing women's earning opportunities, changing their attitude towards violence and building decision-making power within the household, which consequently influences the uptake of maternal healthcare services. ${ }^{3435}$ Moreover, studies from Nepal, ${ }^{11}$
Bangladesh $^{34}$ and India ${ }^{32}$ showed that as an educated household head with economic solvency and possessing decision-making power on healthcare, women had significantly higher chances of receiving proper ANC services than those who did not.

The present study has several strengths. Previous efforts to evaluate prenatal care through indicators of ANC have only considered the opportunity and/or frequency of care. ${ }^{10111334}$ Again, those studies were based on estimating the coverage of each indicator separately or considering different thresholds for each of the components (eg, recommended ANC visits), which represented a barrier for international comparisons. The approach depicted in this study is more comprehensive and combines all the available indicators (in DHS). This measure is internationally comparable and allows the nationwide identification of women who receive frequent, sufficient and appropriate care. By identifying women who received each type of ANC, this study can be used to show the existing gaps in coverage among different socioeconomic groups. Moreover, this nationally representative study was conducted rigorously using standard protocols and trained data collectors with pretesting, which made our findings more reliable and valid. Nonetheless, our study has several limitations. First, because of using DHS data set across countries, adequacy of ANC was possible to measure including only three indicators and thus be 
seen as a starting point for the assessment of adequacy rather than a definitive measure. Second, WHO (2016) ${ }^{19}$ has recently updated the ANC guidance by increasing the number of ANC visits to a minimum of eight contacts with a trimester-wise timeline during pregnancy. But, most of the global, regional and comparable country-reported data, such as DHS, are only available for the previous recommendation (minimum of four visits), and data regarding timing are not available in all countries. ${ }^{9} 1836$ Third, the findings may introduce recall biases that may not correctly present the health-seeking behaviours of mothers. Finally, due to data limitation, this study mainly focused on the relationship of adequate ANC with WESES but could not address the confounding effects of other potential factors (cost of care, availability, and accessibility of health facilities, equity in health service delivery, the timing of ANC visits and knowledge and attitudes towards modern healthcare services) which may act as a barrier for achieving recommended level of adequate ANC.

\section{CONCLUSION}

Adequacy of ANC services was found to be worst in Afghanistan, followed by Bangladesh, compared with the other SA countries. Elevating WES, as well as establishment of necessary healthcare centres in remote areas, is essential to ensure sustainable adequacy of ANC services in SA. A comprehensive understanding through the identified risk factors and incorporating them into short and long-term strategies would help improving this present scenario of the adequacy of ANC services. Special surveillance systems, incentives and proper accommodation arrangements for skilled healthcare providers of rural areas would make them obliged to provide appropriate ANC services to the underprivileged and poor pregnant women. Again, despite being poor, women received adequate ANC due to their high empowerment status. So, national health policies should incorporate and address inequalities in WES, as investments in the empowerment may yield high returns that accrue to individuals, families and societies at large. Additionally, in an economically deprived region like SA, women's higher empowerment level could play an important role to gain full access to adequate ANC services. Therefore, promoting women's empowerment level as well as nationwide awareness programme regarding the necessity of ANC during pregnancy would create demand for higher quality ANC services among SA women.

\section{Author affiliations}

${ }^{1}$ Department of Public Health and Informatics, Bangabandhu Sheikh Mujib Medical University, Dhaka, Bangladesh

${ }^{2}$ Department of Global Health Policy, Faculty of Medicine, The University of Tokyo Graduate School of Medicine, Bunkyo-ku, Tokyo, Japan

${ }^{3}$ Division of Prevention, Center for Public Health Science, National Cancer Center Japan, Chuo-ku, Tokyo, Japan

${ }^{4}$ Research Center for Child Mental Development, Hamamatsu University School of Medicine, Hamamatsu, Shizuoka, Japan

${ }^{5}$ United Graduate School of Child Development, Osaka University, Kanazawa University, Hamamatsu University School of Medicine, Chiba University and University of Fukui, Osaka, Japan
Acknowledgements We are thankful to the MEASURE DHS for serving us with the latest data set. A special thanks and heart full of gratitude goes to Thomas W Pullum, PhD (Director of Research, Demographic and Health Surveys Program, ICF) for his unconditional help and consultation during conceptualisation of research. 'Thank you, Tom, for your unconditional help to your pen-friend.'-Anik

Contributors AIA conceptualised and designed the study. MSR supervised the study. MRI, MSR and AIA curated and analysed the data. AIA interpreted the data, wrote the original manuscript, reviewed, and edited the final manuscript with important intellectual input from all the authors. All authors approved the final manuscript for submission.

Funding The authors have not declared a specific grant for this research from any funding agency in the public, commercial or not-for-profit sectors.

Competing interests None declared.

Patient consent for publication Not required.

Ethics approval Ethical approval for each Demographic and Health Survey is taken from the ICF Institutional Review Board as well as by the respective review boards in the host countries. More details of such ethical approval can be found in the DHS Program website (https://dhsprogram.com/).

Provenance and peer review Not commissioned; externally peer reviewed.

Data availability statement Data are available in a public, open-access repository. The relevant data were obtained from MEASURE DHS and are available from the Demographic Health Surveys Program (Afghanistan Standard DHS, 2015, https://dhsprogram.com/what-we-do/survey/survey-display-471.cfm; Bangladesh Standard DHS, 2014, https://dhsprogram.com/data/dataset/Bangladesh_SPA_ 2014.cfm?flag=0; India Standard DHS, 2015-2016, https://dhsprogram.com/ what-we-do/survey/survey-display-355.cfm; Nepal Standard DHS, 2016, https:// dhsprogram.com/data/dataset/Nepal_Standard-DHS_2016.cfm?flag=0; Pakistan Standard DHS, 2017-2018, https://dhsprogram.com/publications/publication-fr354dhs-final-reports.cfm).

Supplemental material This content has been supplied by the author(s). It has not been vetted by BMJ Publishing Group Limited (BMJ) and may not have been peer-reviewed. Any opinions or recommendations discussed are solely those of the author(s) and are not endorsed by BMJ. BMJ disclaims all liability and responsibility arising from any reliance placed on the content. Where the content includes any translated material, BMJ does not warrant the accuracy and reliability of the translations (including but not limited to local regulations, clinical guidelines, terminology, drug names and drug dosages), and is not responsible for any error and/or omissions arising from translation and adaptation or otherwise.

Open access This is an open access article distributed in accordance with the Creative Commons Attribution Non Commercial (CC BY-NC 4.0) license, which permits others to distribute, remix, adapt, build upon this work non-commercially, and license their derivative works on different terms, provided the original work is properly cited, appropriate credit is given, any changes made indicated, and the use is non-commercial. See: http://creativecommons.org/licenses/by-nc/4.0/.

ORCID iD

Asibul Islam Anik http://orcid.org/0000-0002-6885-2256

\section{REFERENCES}

1 Heredia-Pi I, Servan-Mori E, Darney BG, et al. Measuring the adequacy of antenatal health care: a national cross-sectional study in Mexico. Bull World Health Organ 2016;94:452-61.

2 WHO, UNICEF, UNFPA, World Bank Group and UNDP. Maternal mortality: Levels and trends 2000 to 2017 [Internet]. 104 p, 2019. Available: http://www.who.int/reproductivehealth/publications/ maternal-mortality-2000-2017/en/ [Accessed 30 Apr 2020].

3 World Health Organization. Strategies towards ending preventable maternal mortality (EPMM), 2015.

4 Adeyinka DA, Olakunde BO, Muhajarine N. Evidence of health inequity in child survival: spatial and Bayesian network analyses of stillbirth rates in 194 countries. Sci Rep 2019;9:1-11.

5 Say L, Chou D, Gemmill A, et al. Global causes of maternal death: a who systematic analysis. Lancet Glob Health 2014;2:e323-33.

6 Pervin J, Rahman SM, Rahman M, et al. Association between antenatal care visit and preterm birth: a cohort study in rural Bangladesh. BMJ Open 2020;10:e036699.

7 World Health Organization. Reproductive health indicators : guidelines for their generation, interpretation and analysis for global 
monitoring, 2006. Available: https://apps.who.int/iris/handle/10665/ 43185

8 World Health Organization. WHO antenatal care randomized trial : manual for the implementation of the new model [Internet]. World Health Organization, 200237 p. Available from. Available: https:// apps.who.int/iris/handle/10665/42513 [Accessed 01 May 2020].

9 UNICEF. Antenatal care [Internet], 2019. Available: https://data. unicef.org/topic/maternal-health/antenatal-care/ [Accessed 01 May 2020].

10 Islam MM, Masud MS. Determinants of frequency and contents of antenatal care visits in Bangladesh: assessing the extent of compliance with the who recommendations. PLoS One 2018;13:e0204752.

11 Joshi C, Torvaldsen S, Hodgson R, et al. Factors associated with the use and quality of antenatal care in Nepal: a population-based study using the demographic and health survey data. BMC Pregnancy Childbirth 2014;14:94.

12 Pratley P. Associations between quantitative measures of women's empowerment and access to care and health status for mothers and their children: a systematic review of evidence from the developing world. Soc Sci Med 2016;169:119-31.

13 Arsenault C, Jordan K, Lee D, et al. Equity in antenatal care quality: an analysis of 91 national household surveys. Lancet Glob Health 2018;6:e1186-95.

14 Pambè MW, GNOUMOU/THIOMBIANO B, Kaboré I. Relationship between women's socioeconomic status and empowerment in Burkina Faso: A focus on participation in decision-making and experience of domestic violence. APS 2014;28:1146-56.

15 Croft TN, Marshall AMJ, Allen CK. Guide to DHS Statistics, DHS-7 [Internet]. Rockville, Maryland, USA: ICF, 2018. https://dhsprogram. $\mathrm{com} / \mathrm{pubs} / \mathrm{pdf} / \mathrm{DHSG} 1 / \mathrm{Guide}$ to DHS Statistics DHS-7.pdf

16 ICF International. Demographic and Health Survey Interviewer's Manual [Internet]. Rockville, Maryland, U.S.A: ICF, 2019. https://www. dhsprogram.com/pubs/pdf/DHSM1/DHS7-Interviewer's-Manual-EN12Feb2019-DHSM1.pdf

17 DHS Program. The DHS Program - Protecting the Privacy of DHS Survey Respondents [Internet], 2018. Available: https://dhsprogram. com/What-We-Do/Protecting-the-Privacy-of-DHS-SurveyRespondents.cfm [Accessed 11 Aug 2018].

18 Croft TN, Marshall AMJ, Allen CK. Guide to DHS Statistics, DHS7 [Internet]. Rockville, Maryland, USA: ICF, 2018. https://www. dhsprogram.com/pubs/pdf/DHSG1/Guide_to_DHS_Statistics_DHS7.pdf

19 WHO. WHO recommendations on antenatal care for a positive pregnancy experience [Internet]. 152 p, 2016. Available: http://www. who.int/reproductivehealth/publications/maternal_perinatal_health/ anc-positive-pregnancy-experience/en/ [Accessed 26 Apr 2020].

20 Prata N, Fraser A, Huchko MJ, et al. Women's EMPOWERMENT and family planning: a review of the literature. J Biosoc Sci 2017;49:713-43.

21 Sanawar SB, Islam MA, Majumder S, et al. Women's EMPOWERMENT and intimate partner violence in Bangladesh: investigating the complex relationship. J Biosoc Sci 2019;51:188-202.
22 et alTuladhar S, Khanal KR KCL, Ghimire PK. Women's empowerment and spousal violence in relation to health outcomes in Nepal: Further Analysis of the 2011 Nepal Demographic and Health Survey, 2013. Available: https://dhsprogram.com/ publications/publication-fa77-further-analysis.cfm [Accessed $25 \mathrm{Apr}$ 2020].

23 Asaolu IO, Alaofè H, Gunn JKL, et al. Measuring women's Empowerment in sub-Saharan Africa: exploratory and confirmatory factor analyses of the demographic and health surveys. Front Psychol 2018;9:994.

24 Do M, Kurimoto N. Women's empowerment and choice of contraceptive methods in selected African countries. Int Perspect Sex Reprod Health 2012;38:023-33.

25 Hair JF, Black WC, Babin BJ. Multivariate data analysis. Vol. 5. Prentice hall Upper Saddle River, NJ, 1998.

26 Rutstein SO, Johnson K. The DHS wealth index [Internet] (DHS Comparative Reports No. 6). Calverton, Maryland, USA: ORC Macro, 2004. http://dhsprogram.com/pubs/pdf/CR6/CR6.pdf

27 Ahmed S, Creanga AA, Gillespie DG, et al. Economic status, education and empowerment: implications for maternal health service utilization in developing countries. PLoS One 2010;5:e11190.

28 Chol C, Negin J, Agho KE, et al. Women's autonomy and utilisation of maternal healthcare services in 31 sub-Saharan African countries: results from the demographic and health surveys, 2010-2016. BMJ Open 2019;9:e023128.

29 Wang W, Alva S, Wang S. Levels and trends in the use of maternal health services in developing countries, 2011. Available: https:// dhsprogram.com/publications/publication-cr26-comparative-reports. cfm [Accessed 29 Jun 2020].

$30 \mathrm{WHO}, \mathrm{UNICEF}$. Antenatal care in developing countries. Promises achievements and missed opportunities: an analysis of trends, levels and differentials [Internet] 37 p, 2003. Available: http://www. who.int/reproductivehealth/publications/maternal_perinatal_health/ 9241590947/en/ [Accessed 29 Jun 2020].

31 N.R Ravindra Deyshappriya. Poverty in South Asia: The long view [Internet]. South Asia @ LSE, 2019. Available: https://blogs.Ise.ac. uk/southasia/2019/04/09/poverty-in-south-asia-the-long-view/ [Accessed 29 Jun 2020].

32 Rani M, Bonu S, Harvey S. Differentials in the quality of antenatal care in India. Int J Qual Health Care 2008;20:62-71.

33 Merrell LK, Blackstone SR. Women's Empowerment as a Mitigating Factor for Improved Antenatal Care Quality despite Impact of 2014 Ebola Outbreak in Guinea. Int J Environ Res Public Health 2020;17:8172.

34 Pulok MH, Sabah MN-U, Uddin J, et al. Progress in the utilization of antenatal and delivery care services in Bangladesh: where does the equity gap lie? BMC Pregnancy Childbirth 2016;16:200.

35 Matsumura M, Gubhaju BB. Women's status, household structure and the utilization of maternal health services in Nepal. Asia Pac Popul J 2001;16:23-44.

36 NIPORT/Bangladesh NI of PR and T-, Associates M and, International ICF. Bangladesh demographic and health survey 2014, 2016. Available: http://dhsprogram.com/publications/publicationFR311-DHS-Final-Reports.cfm [Accessed 26 Mar 2018] 